\title{
Supratentorial low grade astrocytoma: prognostic factors, dedifferentiation, and the issue of early versus late surgery
}

\author{
M L C van Veelen, C J J Avezaat, J M Kros, W van Putten, Ch Vecht
}

\begin{abstract}
Background-A retrospective study of patients with low grade astrocytoma was carried out because the best management of such patients remains controversial. Prognostic factors were identified by multivariate analysis. Special attention was paid to the effect of extent and timing of surgery.
\end{abstract}

Methods-Ninety patients with low grade astrocytoma were studied. Seventy two patients had resective surgery, 15 had a diagnostic biopsy only, and three patients had resective surgery after initial biopsy.

Results-Significant prognostic factors for survival were age, preoperative neurological condition, epilepsy as the single sign, extent of surgery, and histology. The extent of surgery was highly significant on univariate analysis $(p=0.002)$; however, after correction for age and preoperative symptoms this was considerably reduced $(p=0.04)$. A subgroup of 30 patients with epilepsy as their single presenting symptom was identified. Thirteen of these patients were treated immediately after diagnosis, whereas the other 17 patients were initially followed up and treated only after clinical or radiological progression. Survival in both groups was identical (63\% survival rate after five years) and much better than survival for the whole group ( $27 \%$ survival rate after five years). Malignant dedifferentiation was observed in $25(70 \%)$ of 36 patients who were reoperated, after a median period of 37 months. This period was 41 months for the subgroup of patients with epilepsy only and 28 months for the remaining patients.

Conclusions-Due to the retrospective nature of the study only restricted conclusions can be drawn. Low grade glioma with epilepsy as the single symptom has a much better prognosis than if accompanied by other symptoms. This prognosis is not influenced by the timing of surgery. It seems, therefore, safe to defer surgery until clinical or radiological progression in low grade glioma with epilepsy only.

\section{(F Neurol Neurosurg Psychiatry 1998;64:581-587)}

Keywords: low grade astrocytoma; prognostic factors; dedifferentiation; deferred surgery

The best management of patients with low grade astrocytoma remains controversial. ${ }^{1-3}$
The timing of surgery, the extent of surgery, and the need for radiotherapy continue to be a matter of discussion despite many retrospective studies.

The timing of surgery became an issue after the introduction of CT. Due to its better sensitivity, CT disclosed lesions in an earlier stage than scintigraphy. Early diagnosis by CT raised the question as to whether early operation in patients with no or minor neurological deficit would benefit the duration and quality of survival, considering the rather benign course of the disease and the risk of postoperative neurological damage. The fact that in most patients extensive surgery is a favourable prognostic factor ${ }^{4-10}$ is often used as an argument in favour of early surgery. However, the two retrospective studies that specifically considered the issue of deferral of therapy did not show clear differences in survival nor in the rate of malignant transformation between early or late surgery. ${ }^{11}{ }^{12} \mathrm{We}$ analysed prognostic factors in 90 patients with low grade astrocytoma. Treatment strategy changed over time due to the increasing availability of CT, introduction of stereotactic biopsy, and fluctuation in opinion on the preferred management. These changes resulted in two treatment strategies for the 30 patients with epilepsy as their single presenting sign. One group was treated early and one group was initially followed up and treated after clinical or radiological progression. We studied the factors that influenced the choice and outcome of either strategy.

\section{Materials and methods}

This study comprised all 90 patients with a histologically confirmed low grade astrocytoma who had their first operation or biopsy between 1975 and 1989. Patients who were less than 18 years of age at operation were excluded. Only supratentorial tumours were included. No patient was lost to follow up.

Age, sex, presenting symptoms, preoperative duration of symptoms, location, and CT characteristics of the lesion, preoperative and postoperative performance status, type, and extent of surgery, as well as complications, histology, radiotherapy, and survival were taken from the patients' charts.

The performance status was scored before operation and at discharge from the hospital using a neurological function classification on a four point scale ${ }^{13}$ :

$0=$ able to work, with only slight or no neurological deficit (includes mild headache, epileptic insults controlled with anticonvulsants, minimal aphasia, minimal hemianopia, 
slight hemiparesis, sensory disturbances); $1=$ able to be at home, with minor neurological deficit (includes headache, epileptic insults, some apathy permitted, mild aphasia, hemianopia, hemiparesis grade 4 (MRC scale)); $2=$ requiring hospital admission, with major neurological findings (includes organic brain syndrome, severe headache, aphasia, hemiparesis grade 2-3, sphincter disturbances, wheelchair dependent); $3=$ requiring hospital admission and in serious physical or neurological state (includes diminished consciousness or coma, severe aphasia, hemiplegia (0-1), severe ataxia, bedridden more than $50 \%$ of the time).

All charts contained a fairly extensive report on the operation, which allowed us to estimate the extent of tumour resection using the following scale: $1=$ stereotactic biopsy (performed since 1983); $2=$ partial resection (estimated tumour removal $<75 \%) ; 3=$ gross total removal (estimated tumour removal $\geqslant 75 \%$ ).

Postoperative CT to more reliably estimate the extent of tumour removal was not available on a routine basis.

All histological slides were reviewed by the neuropathologist. Low grade astrocytoma according to World Health Organisation (WHO; 1993) criteria as well as low grade oligodendrogliomas and mixed types were included. Pilocytic astrocytomas and low grade gliomas in the posterior fossa were excluded.

Available options for therapy were early surgery, biopsy only, or deferral of surgery, with or without initial diagnostic biopsy. Deferral of surgery or the "wait and see" policy was considered as a feasible option only for patients with epilepsy as the single presenting symptom. In the case of a "wait and see" policy, surgery was performed after clinical or radiological progression. In an attempt to identify a potential bias the arguments for or against either therapy choice were recorded from the patient's charts. In most cases the reasoning as to which treatment was chosen was not extensively described. Therefore we first defined possible arguments and then scored them as "present" or "not present", further adding arguments as we encountered them in the charts.

In general, patients were reoperated on if recurrence occurred more than one year after initial surgery. In case of dedifferentiation of tumour, radiotherapy was applied. Radiotherapy was also administered in patients with an inoperable recurrence.

The follow up period of this study was censored at 31 December 1993. At that time the patient's condition was recorded as either alive, dead, or as hospital mortality (death within 30 days after surgery, from surgical or medical complications). Date of recurrence was scored at the time of increase of symptoms or of radiological progression of the tumour after surgery.

Survival curves and mortality rates were calculated according to the actuarial method of Kaplan and Meier. Greenwood's formula was applied to calculate the standard error (SE) of the survival rate estimates. In addition to the overall survival duration we calculated the pro- gression free survival, which was defined as the survival until the first date of recurrence or death. For the survival analysis the overall survival duration and the progression free survival were measured from the day of first surgery. However, when comparing the survival from immediate and deferred surgery in the subgroup of patients with only epilepsy, survival duration was measured from the day of the first positive scan, because this was considered, retrospectively, as the day that the decision was made between immediate or deferred surgery.

The Cox proportional hazards model was used to determine the patient and tumour characteristics that were most related to prognosis, measured by the duration of survival and the progression free survival. All factors mentioned in table 3 as well as contrast enhancement on CT and duration of symptoms were fed into the Cox univariate analysis. The associated log likelihood ratio test was applied to identify differences or trends in survival and progression free survival between groups, in multivariate analysis.

\section{Results}

PATIENTS' CHARACTERISTICS

Ninety patients met all inclusion criteria. Table 1 lists the patients' characteristics preoperatively. Mean age was 41 (SD 12) years. Sixty one patients $(68 \%)$ presented with epilepsy and in 30 of them epilepsy was the only symptom. Most tumours were located in one or more cerebral lobes. One was located in the thalamus and there was one ventricular tumour. Six lesions were bilateral. Table 1 gives the CT characteristics. Interestingly, a considerable number of CT lesions (34\%) showed contrast enhancement. Seven patients were initially diagnosed by scintigraphy.

TREATMENT

Of the $30(33 \%)$ patients with epilepsy as the single presenting symptom 13 were selected for

Table 1 Patient characteristics

\begin{tabular}{lc}
\hline & $\begin{array}{c}\text { Patients } \\
(n=90)\end{array}$ \\
& $n$ \\
\hline Mean age (y) & 41 \\
$\leq 40$ & 45 \\
$>40$ & 45 \\
Sex: & 34 \\
F & 34 \\
M & 56 \\
Presenting symptoms: & \\
Epilepsy & 61 \\
Epilepsy only & 30 \\
Headache & 28 \\
Papiloedema & 17 \\
Aphasia & 7 \\
Hemiparesis & 19 \\
Visual defect & 4 \\
Confusion & 11 \\
Coma & 3 \\
Ataxia & 2 \\
Position of tumour: & \\
Right & 51 \\
Left & 33 \\
Bilateral & 6 \\
CT characteristics: & \\
Hypodensity & 71 \\
Mixed density & 12 \\
Enhancement & 39 \\
Cysts & 9 \\
Calcification & 9 \\
\hline
\end{tabular}


Table 2 Treatment

\begin{tabular}{ll}
\hline & $\begin{array}{c}\text { Patients } \\
(n=90) n\end{array}$ \\
\hline Preoperative performance status: & \\
0 & 31 \\
1 & 29 \\
2 & 24 \\
3 & 6 \\
Delay from positive scan (months): & \\
Mean (SD) & 7,20 \\
Range & $0-138$ \\
Median & 1 \\
Number & 90 \\
Symptoms at surgery: $\dagger$ & \\
Epilepsy & 21 \\
Epilepsy + other & 41 \\
No epilepsy & 28 \\
Number of symptoms: & \\
1 & 29 \\
2 & 30 \\
23 & 31 \\
Extent of surgery: & \\
Biopsy & 18 \\
<75\% & 59 \\
$>75 \%$ & 13 \\
Histology: & \\
Astrocytoma & 72 \\
Oligodendro & 14 \\
Mixed type & 4 \\
\hline
\end{tabular}

*Time between first positive scan and first operation. +Note that these are the symptoms immediately before operation, nine of the 30 patients with initially only epilepsy developed additional symptoms later.

immediate surgery, whereas for the remaining 17 patients a "wait and see" policy was chosen. Eventually, all patients in the "wait and see" group underwent surgery. The median interval between the first positive scan and surgery was 14 months (mean 29 months), with a maximum of 138 months. The median interval for the group with immediate surgery was one month (mean two months).

The remaining 60 patients who presented with neurological deficit (with or without epilepsy) were operated on without actual

Table 3 Overall survival (OS) and progression free survival (PFS) probabilities at 5 years

\begin{tabular}{|c|c|c|c|c|}
\hline & $n$ & $\begin{array}{l}\text { Alive at } 5 \\
\text { years }\end{array}$ & $O S(S E)(\%)$ & $P F S(S E)(\%)$ \\
\hline $\mathrm{n}$ & 90 & 23 & $27(5)$ & $19(4)$ \\
\hline Age (y): & & & $\star \star$ & $\star \star$ \\
\hline$\leqslant 40$ & 45 & 14 & $35(7)$ & $22(5)$ \\
\hline $40-50$ & 28 & 7 & $25(8)$ & $21(8)$ \\
\hline$>50$ & 17 & 2 & $12(8)$ & $6(6)$ \\
\hline Performance status: & & & $\star \star \star \star$ & $\star \star \star \star$ \\
\hline 0 & 31 & 21 & $44(9)$ & $35(9)$ \\
\hline 1 & 29 & 10 & $35(9)$ & $17(7)$ \\
\hline 2 & 24 & 0 & 0 & 0 \\
\hline 3 & 6 & 1 & $17(15)$ & $17(15)$ \\
\hline Location: & & & $\star \star$ & $\star$ \\
\hline $\mathrm{R}$ & 51 & 18 & $35(7)$ & $29(6)$ \\
\hline $\mathrm{L}$ & 33 & 5 & $20(7)$ & $6(4)$ \\
\hline$M$ & 6 & 0 & 0 & 0 \\
\hline Symptom: & & & $\star \star \star \star$ & $\star \star \star \star$ \\
\hline Epilepsy & 21 & 11 & $61(11)$ & 47 (11) \\
\hline Epilepsy + other & 41 & 7 & $17(6)$ & $15(6)$ \\
\hline No epilepsy & 28 & 5 & $18(7)$ & $4(4)$ \\
\hline Symptoms (n): & & & $\star \star \star$ & $\star \star \star$ \\
\hline 1 & 29 & 12 & $48(9)$ & $37(9)$ \\
\hline 2 & 30 & 9 & $30(8)$ & $13(6)$ \\
\hline 3 & 31 & 2 & $7(4)$ & $7(4)$ \\
\hline Histology: & & & $\star \star$ & $\star$ \\
\hline Astro & 72 & 14 & $22(5)$ & $17(4)$ \\
\hline Oligo & 14 & 8 & $57(13)$ & $29(12)$ \\
\hline Mixed & 4 & 1 & $25(22)$ & $25(22)$ \\
\hline Extent of surgery: & & & 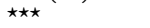 & 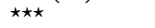 \\
\hline Biopsy & 18 & 5 & $33(11)$ & $28(11)$ \\
\hline$<75 \%$ & 59 & 10 & $18(5)$ & $10(4)$ \\
\hline$>75 \%$ & 13 & 8 & $62(14)$ & $46(14)$ \\
\hline
\end{tabular}

${ }^{\star} \mathrm{p}<0.05 ;{ }^{\star \star} \mathrm{p}<0.01 ;{ }^{\star \star \star} \mathrm{p}<0.001$ by $\log$ likelihood ratio tests.

Extent of surgery: $>75 \% v$ rest.

Histology: astro $v$ oligo.

Age, performance status, number of symptoms, by test for trend deferral, one month (median) after the first positive CT.

At the time of surgery one third of the patients had a good (grade 0), one third an intermediate (grade 1), and one third a poor performance status (grade 2 or 3 ). The performance status improved after operation in 27 patients: $28 \%$ of the patients with preoperative performance status grade 1 and $63 \%$ of the patients with preoperative performance status grade 2 or 3 . Four patients had a lower performance status after operation.

The surgical procedure was restricted to a biopsy in 18 patients. Three of them underwent decompressive surgery later (after nine, 21 , and 70 months). Seventy two patients had a more or less extensive tumour resection. Only in 13 of these 72 patients an estimated resection of more than $75 \%$ was achieved. Nine patients had medical complications and three had surgical complications. Four patients died within one month of surgery.

Thirty six patients were reoperated on at least once. Fifteen of these patients had a third operation and four a fourth operation. In another six patients the symptomatic regrowth was primarily (mono)cystic and they only had a drain implanted in the cyst.

Thirty one patients received radiotherapy at recurrence either because the recurrent tumour was deemed inoperable or because histology at reoperation showed dedifferentiation to a higher grade of malignancy.

HISTOLOGY

In most patients histology disclosed a low grade astrocytoma (table 2). (None showed mitotic activity, cell polymorphism, or necrosis, only moderate cell density was accepted.) Oligodendroglioma and mixed types of tumours occurred less often. Thirty six patients were reoperated at least once. In 25 of them a higher grade of malignancy was diagnosed at either the second, third, or fourth operation. This occurred after a median period of 37 months (actuarial estimate; range 3-84 months) for the whole group, 41 months for the patients with epilepsy only, and 28 months for the other patients. Within the group of patients with epilepsy only this period was 40 and 43 months, respectively for the deferred and immediate treatment group. In 11 of the 36 reoperated patients no dedifferentiation was noted. Their reoperation was after a median period of 19 months.

\section{SURVIVAL AND PROGRESSION AFTER SURGERY} Of the 90 patients 82 showed progression during follow up and 75 patients died (table 3 ). The overall survival probability for the whole group was $27 \%$ (SE 5) at five years and $14 \%$ (SE 4) after 10 years. The median survival time was 36 months.

Table 3 shows the five year overall survival and progression free survival probabilities for subgroups with different features; figure 1 shows the corresponding survival curves. The patterns in overall survival and progression free survival were similar. Favourable characteristics were younger age (fig 1A), epilepsy as single 

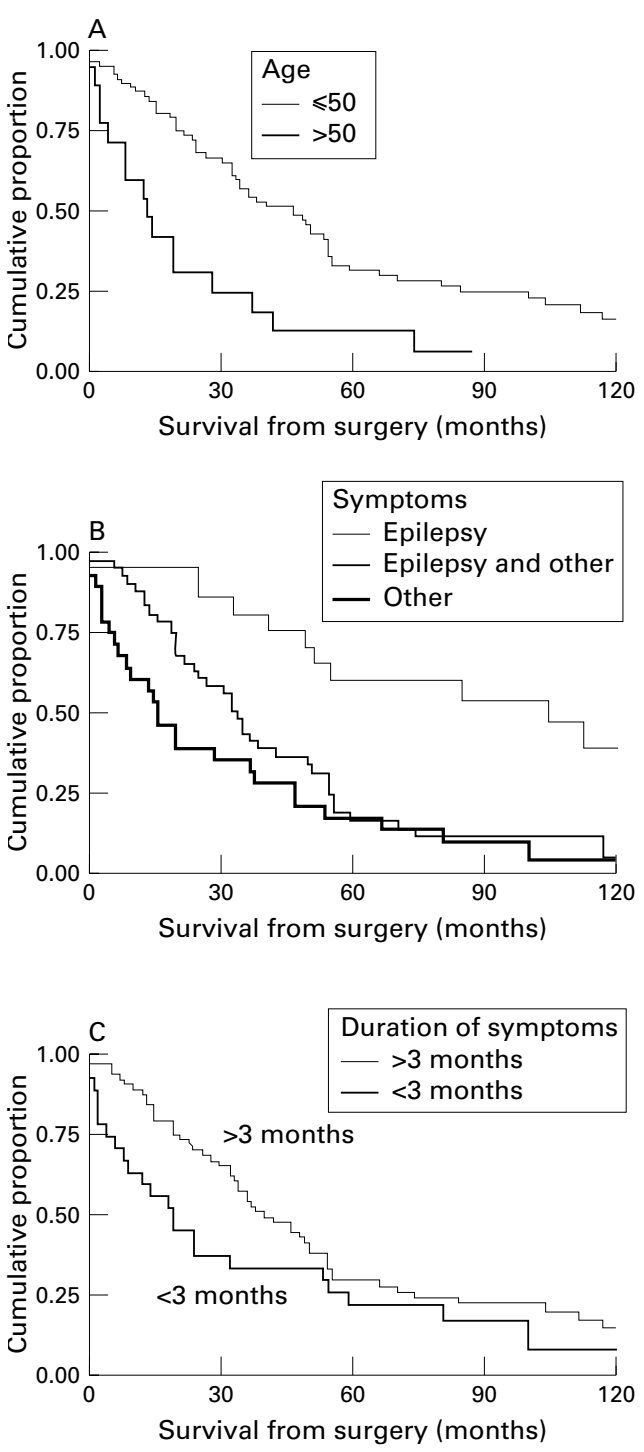

Figure 1 (A) Survival curves for patients aged $>50$ or $\leqslant 50$. (B) Survival curves for patients who immediately before surgery had only epilepsy, epilepsy and other symptoms, or only other symptoms. (C) Survival curves for patients who before surgery had had symptoms for $<3$ months or $>3$ months.

presenting symptom (fig 1B), and oligodendroglioma (compared with astrocytoma). The absence of contrast enhancement was not identified as a favourable characteristic. The duration of preoperative symptoms did not show a significant trend with overall survival or progression free survival over the whole follow up, but fig $1 \mathrm{C}$ shows that the death rate in the first years was much higher in the group of patients with a preoperative duration of symptoms<three months. This analysis, however, should primarily be regarded as exploratory because most of the subgroups are small and some of the characteristics are interrelated.

Multivariate Cox regression analysis identified age over 50 years (relative death rate $(\mathrm{RDR})=2.5, \mathrm{p}=0.002)$, absence of epilepsy ( $R D R=3.5, p=0.001$ ), and three or more symptoms before surgery $(\mathrm{RDR}=2.2$, $\mathrm{p}=0.007$ ) as the most important factors. Given these factors patients with oligodendrogliomas showed a lower death rate than the patients with astrocytomas. However, the difference was not significant $(\mathrm{RDR}=0.5, \mathrm{p}=0.06)$. Grade of malignancy, preoperative performance status, nor location of the lesion showed a significant association with survival, given age, and symptoms.

The patients whose tumours could be decompressed extensively fared much better than the patients who had just a biopsy or a small decompression of their tumour. However, the differences in survival related to extent of surgery seem to be partly evidence of selection. The 13 extensive resections were performed primarily in younger patients (12 patients $<50$ years) presenting with epilepsy (eight patients with epilepsy only, three patients with epilepsy and one other symptom) who had a right sided tumour (10 of 13). Also, a large resection occurred more often among patients with an oligodendroglioma (five of 14) than among patients with an astrocytoma (seven of 72). Without adjustment for age and symptoms the difference in survival between patients with a large resection and the rest was highly significant $(p=0.002)$, but this was reduced considerably after adjustment for age and symptoms $(\mathrm{p}=0.04)$.

Radiotherapy was not evaluated as a prognostic factor, because the indications for radiotherapy were variable, and would probably produce a highly biased value.

“WAIT AND SEE" VERSUS IMMEDIATE SURGERY The patients who were operated on immediately were compared with those with a "wait and see" policy. Figure 2 shows the overall survival, calculated from the date of the first positive CT or scintigraphy, for both treatment options in the subgroup epilepsy only (the overall survival for the other patients with neurological deficit is shown as well). There is no clear difference between both treatment groups; they have a pooled five year overall survival of 63 (SE 9)\% and a 10 year overall survival of 32 (SE 9)\%. The estimate of the relative death rate in the immediate surgery group compared with the "wait and see" group was 1.02 (95\% confidence interval $0.4-2.5$ ). This result has to be interpreted cautiously. We tried to identify potential biases, firstly, in the arguments for either therapy found in the charts and secondly, in different patient characteristics of both treatment groups. Arguments to choose the "wait and see" option were (more than one argument for each patient possible): reluctance of the patient to surgery (one) no neurological deficit (15), diffuse and extensive hypodensity on CT (10), and lesion located in eloquent areas (seven). Reasons to operate after initial delay were the patient's wish (one), incontrollable seizures (two), neurological deficit (10), progression on CT (seven), and acute tumour haemorrhage with signs of herniation (one). Arguments for early surgery were the patient's wish (one), the need for histological verification (six), contrast enhancement on CT (three), supposed arachnoid cyst on CT (one), and location favourable to surgery (10). Clearly, the reasons for deciding on either treament strategy are numerous 


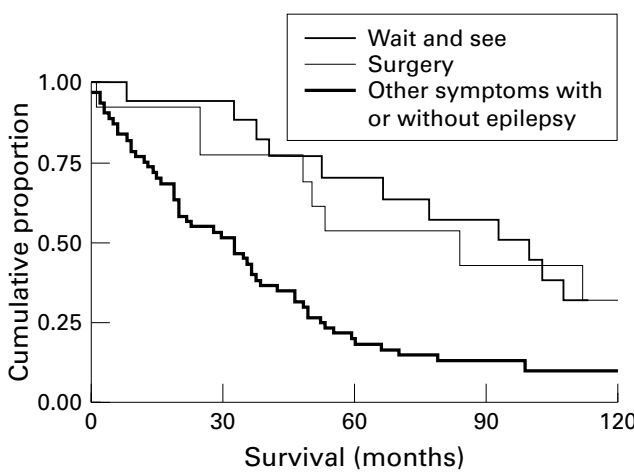

Figure 2 The "wait and see" and surgery groups had epilepsy only at referral. The group with other symptoms presented with or without epilepsy.

Table 4 Patient characteristic differences in group with epilepsy only

\begin{tabular}{lcc}
\hline & "Wait and see” & Surgery \\
\hline Age & 41 & 33 \\
Radiotherapy: & 9 & 1 \\
$\quad$ Yes & 8 & 12 \\
$\quad$ No & & \\
Extent of surgery: & 6 & 0 \\
$\quad$ Biopsy & 8 & 7 \\
$\quad$ Small & 3 & 6 \\
$\quad$ Gross & 0 & 9 \\
Astrocytoma & 6 & 3 \\
Oligo & & \\
Position of tumour: & 8 & 12 \\
$\quad$ Right & 9 & 1 \\
$\quad$ Left & & \\
\hline
\end{tabular}

and diverse. Most important arguments are firstly the operability of the lesion-that is, location of the lesion and diffuse growth pattern, and secondly the expected malignancy. These arguments are partially reflected in the differences in the patients' characteristics (table 4). Early treated patients were younger (mean age $33 v 41$ years), had more right sided lesions (12 of $13 v$ eight of 17), and were operated on more extensively. Contrast enhancement on CT occurred slightly more often in the early treated group (four of 13 versus three of 17). Thus the early treated group tended to have the most favourable patient characteristics for survival. Therefore, the lack of a better survival rate in the early treated group seems not to be produced by a preponderance of unfavourable patient characteristics in this group.

Table 5 Significant prognostic factors and effect of treatment reported in the literature

\begin{tabular}{|c|c|c|c|c|c|}
\hline & Patients $n$ & Age & $\begin{array}{l}\text { Preoperative } \\
\text { condition }\end{array}$ & $\begin{array}{l}\text { Extent of } \\
\text { surgery }\end{array}$ & Radiotherapy \\
\hline Laws $e t a l^{4}$ & 461 & + & + & + & + \\
\hline Piepmeier $^{8}$ & 60 & - & + & + & - \\
\hline Medbery et al $l^{7}$ & 50 & + & & $+(>1978)$ & $+^{\star}$ \\
\hline Sofietti et $a l^{6}$ & 85 & - & + & + & - \\
\hline Shaw et $a l^{16}$ & 126 & + & - & - & $+^{\star}$ \\
\hline North et al & 77 & + & + & + & - \\
\hline McGormack et al ${ }^{17}$ & 53 & + & + & & \\
\hline Miralbell et al ${ }^{9}$ & 67 & + & + & $+\$$ & \\
\hline Shibamato et al ${ }^{18}$ & 119 & + & & - & + \\
\hline Janny et $a l^{10} \dagger$ & 58 & - & - & $+\ddagger$ & - \\
\hline This study & 90 & + & + & $+\Phi$ & + \\
\hline
\end{tabular}

^Dose dependent.

†Pilocytic astrocytomas included.

$\ddagger$ Kernohan grade 1 and 2 were subdifferentiated in 3 grades.

§Especially for subtype oligodendroglioma.

TOnly for tumour resection $>75 \%$.

\section{Discussion}

Survival rates and favourable prognostic factors in this study are comparable with series reported previously ${ }^{4-12}$ 14-21 (table 5), although the five year survival rate is lower than in most studies. This could be explained by the relatively high age in this group and the high percentage of small tumour resections.

Practically all studies identify age as a strong independent prognostic factor. Patients under the age of 20 years have a five year survival rate of about $80 \%$, between age $20-40$ the survival rate decreases to about $50 \%$, whereas survival rates for patients above 40 years of age are around $30 \%$.

Extent of surgery is another favourable prognostic factor in most studies. It should be realised, however, that extent of surgery may be subject to inaccuracy and bias. The surgeon's estimation of the size of resection is known to be of limited accuracy. Postoperative CT was not done routinely in most series. Deep seated lesions, older patients, or patients with gross neurological deficit possibly tend to be operated on less extensively. Furthermore, in several studies, ${ }^{4-1015-18}$ operative deaths were not included in their analysis. However, for assessment of the true effects of surgery it is more realistic to include the associated mortality. Reported percentages of death related to surgery in the 1970 s and 80 s vary between $2 \%$ and $9 \%$. Finally, extensive surgery seems to be especially effective in younger patients, ${ }^{416}$ but this finding may have been influenced by the higher number of pilocytic astrocytomas in the younger group. ${ }^{16}$ In considering the effect of extensive surgery, the findings of Vertosick $e t a l^{19}$ are surprising. His patients received radiotherapy after verification of histology by stereotactic biopsy only. $\mathrm{He}$ reported a median survival of 8.2 years in a group of 25 patients. These results, however, can at least partially be explained by the young age of 35 years of his population and the very low percentage of enhancing lesions on CT.

About half of the reports indicate that radiotherapy is a significant prognostic factor. Laws et $a l,{ }^{4}$ Garcia et $a l,{ }^{15}$ and Shaw et $a l^{16}$ found that radiotherapy benefits primarily older patients. Several studies show a favourable effect only for subtotally removed tumours. ${ }^{4} 152022$ Medbery et $a l^{7}$ and Shaw et $a l^{16}$ established a relation between dose of radiation and duration of survival. However, a recent prospective trial failed to find a dose response to 45 versus $59 \mathrm{Gry}^{21}$

In our study the initial symptom of epilepsy only is a stronger prognostic factor than age. Although the preoperative neurological condition is a significant prognostic factor in most studies, epilepsy only was either not identified as a prognostic factor or not evaluated separately. Only Laws et $a l^{4}$ and North et $a l^{5}$ found epilepsy as a favourable prognostic factor on univariate analysis. However, for anaplastic gliomas, epilepsy has been recognised as a favourable prognostic sign in numerous studies. ${ }^{123-26}$ In our opinion patients with epilepsy only should be considered as a separate group within the low grade glioma. They have 
a better prognosis, which, in combination with younger age and intact neurological condition, may well have implications on choosing the best strategy. ${ }^{27}$ The dilemma of early versus late treatment particularly applies to this group. Early surgery entails a certain risk of morbidity and mortality which may be acceptable if it would produce a substantially better survival. Without surgery these patients seem to have, in most cases, several years of good survival quality with epilepsy as their only symptom.

Two retrospective studies ${ }^{11}{ }_{12}$ considered the management of patients with epilepsy and unverified lesions on CT. Early treatment was compared with deferred treatment. Differences in outcome could not be identified, neither in survival nor in the time until malignant dedifferentiation. It is difficult to explain these findings considering that Laws et $a l^{4}$ and many others reported that extensive surgery produces a better survival. However, it may be erroneous to extrapolate extensive surgery to early surgery.

In our study, early versus late treatment in patients with epilepsy only did not show a clear difference in survival calculated from the time of the first CT. However, our results should be interpreted with some caution. Both groups comprise few patients. Patient characteristics in both groups were not evenly distributed, although the equal survival rate for both groups cannot be explained by the presence of favourable patient characteristics in the group with deferred treatment. Finally, patients were selected for this study based on histology and not on CT. Patients with a "wait and see" policy who were later, on initial surgery, found to carry more malignant lesions, are not included in this study. Excluding these patients from the analysis may have favoured the outcome of the "wait and see" group. Although we are aware of the presence of potential bias, we think that our study shows that deferring treatment is safe for patients with "epilepsy only".

Because of the few patients with "epilepsy only" we did not try to identify subgroups, based on additional prognostic factors such as age, for which deferral of treatment would be less or perhaps more appropriate. Based on the known prognostic factors it may be may argued that for the group of patients over 40 years of age a more aggressive approach may be safer. Their prognosis is worse, and although they probably do not benefit as much from extensive surgery they benefit more from radiotherapy than the younger patients. Moreover, about half of the non-enhancing lesions in patients over 45 years of age seemed to have a malignant lesion grade III or IV. ${ }^{28}$ Therefore, tumour verification seems to be warranted in this group either by resective surgery or biopsy, followed by radiotherapy. The younger patients, on the other hand, have a better prognosis, the need for radiotherapy is not clear, and nonenhancing lesions are highly associated with low grade gliomas. Tumour verification would not alter treatment strategy. In these patients a "wait and see" policy is in our opinion fully justified until better figures come available. ${ }^{27} \mathrm{It}$ is necessary, however, to be aware of the risks of deferred therapy such as tumour haemorrhage, rapid and unexpected deterioration, and also incorrectly labelling the patient as inoperable. Resective surgery should be reconsidered in every patient showing clinical or radiological progression.

\section{Conclusion}

Definition of the best policy for low grade astrocytoma should be based on prospective studies. Our study shows that the outcomes after early or late surgery in patients with epilepsy alone are not clearly different and a prospective study comparing both these options seems warranted. Based on our figures we calculated that the minimum size of the study group totals 250 patients to prove a difference in survival of $20 \%$ and 800 patients to prove a difference of $10 \%$. Considering the incidence of low grade glioma and its survival, only a multicentre trial may answer these questions.

1 Cairncross JG, Laperriere NJ. Low-grade glioma. To treat or not to treat? Arch Neurol 1989;46:1238-9.

2 Shapiro WR. Low-grade gliomas: when to treat? Ann Neurol 1992;31:437-8.

3 Shaw SG. Low-grade gliomas: to treat or not to treat? Arch Neurol 1990;47:1138-9.

4 Laws ER, Taylor WF, Clifton MB, et al. Neurosurgical management of low-grade astrocytoma of the cerebral hemispheres. F Neurosurg 1984;61:665-73.

5 North CA, North RB, Epstein JA, et al. Low-grade cerebral astrocytomas. Survival and quality of survival after radiation therapy. Cancer 1990;66:6-14.

6 Sofietti R, Chio A, Giordana MT, et al. Prognostic factors in well differentiated cerebral astrocytomas in the adult. Neurosurgery 1989;24:686-92.

7 Medbery CA, Straus KL, Steinberg SM, et al. Low-grade astrocytomas: treatment results and prognostic variables. Int F Radiat Oncol Biol Phys 1988;15:837-41.

8 Piepmeier JM. Observations on the current treatment of low-grade astrocytic tumors of the cerebral hemispheres. $\mathcal{f}$ Neurosurg 1987;67:177-81.

9 Miralbell R, Balart J, Matias-Guiu X, et al. Radiotherapy for supratentorial low-grade gliomas: results and prognostic factors with special focus on tumour volume parameters. Radiother Oncol 1993;27:112-6.

10 Janny $\mathrm{P}$, Cure $\mathrm{H}, \mathrm{Mohr} \mathrm{M}$, et al. Low grade supratentorial astrocytomas; management and prognostic factors. Cancer 1994;73:1937-45.

11 Smith DF, Hutton JL, Sandeman D, et al. The prognosis of primary intracerebral tumours presenting with epilepsy: primary intracerebral tumours presenting with epilepsy:
the outcome of medical and surgical management. $\mathscr{f}$ Neurol the outcome of medical and surgical man
Neurosurg Psychiatry 1991;54:915-20.

12 Recht LD, Lew R, Smith TW. Suspected low-grade glioma: is deferring treatment safe? Ann Neurol 1992;31:431-6.

13 Borgelt B, Gelber RD, Kramer S, et al. The palliation of brain metastases: final results of the first two studies by the Radiation Therapy Oncology Group. Int $\mathcal{F}$ Radiat Oncol Biol Phys 1980;6:1-9.

14 Scanlon PW, Taylor WF. Radiotherapy of intracranial astrocytomas: analysis of 417 cases treated from 1960 through 1969. Neurosurgery 1979;5:301-8.

15 Garcia DM, Fulling KH, Marks JE. The value of radiation therapy in addition to surgery for astrocytomas of the adult cerebrum. Cancer 1985;55:919-27.

16 Shaw EG, Daumas-Duport C, Scheithauer BW, et al. Radiation therapy in the management of low-grade supratentorial astrocytomas. F Neurosurg 1989;70:853-61.

17 McGormack BM, Miller DC, Budzilovich GN, et al. Treatment and survival of low-grade astrocytoma in adults, 1977-88. Neurosurgery 1992;31:636-42.

18 Shibamato Y, Kitakabu Y, Takahashi M, et al. Supratentorial low-grade astrocytoma: correlation of computed tomography findings with effect of radiotherapy and prognostic variables. Cancer 1993;72:190-5.

19 Vertosick FT, Selker RG, Arewa VC. Survival of patients with well-differentiated astrocytomas diagnosed in the era of computer tomography. Neurosurgery 1991;28:496-501.

20 Fazekas JT. Treatment of grades I and II brain astrocytomas. The role of radiotherapy. Int f Radiat Oncol Biol Phys 1977;2:661-6.

21 Karim A, Maat B, Hatlevoli R, et al. A randomized trial on dose response in radiation therapy of low-grade cerebral dose response in radiation therapy of low-grade cerebral
glioma: European organization for research and treatment glioma: European organization for research and treatment
of cancer (EORTC) study 22844. Int $\mathcal{f}$ Radiat Oncol Biol of cancer (EORTC) st
Phys 1996;36:549-56.

22 Leibel SA, Sheline GE, Ware WM, et al. The role of radiation therapy in the treatment of astrocytomas. Cancer 1975;35:1551-7. 
23 Walker MD, Alexander E, Hunt WE, et al. Evaluation of $\mathrm{BCNU}$ and/or radiotherapy in treatment of anaplastic glioBCNU and/or radiotherapy in trea
mas. $\mathcal{F}$ Neurosurg $1987 ; 24: 686-91$.

24 Winger MJ, MacDonald DR, Cairncross JG. Supratentorial anaplastic gliomas in adults: the prognostic importance of the extent of resection and prior low-grade glioma. $7 \mathrm{Neu}$ rosurg 1989;71:487-93.

25 MRC Brain Tumour Working Party. Prognostic factors for high-grade malignant glioma: development of a prognostic index. F Neurooncol 1990;9:47-55.
26 Scott GM, Gibberd FB. Epilepsy and other factors in the prognosis of gliomas. Acta Neurol Scand 1980;61:227 39 .

27 Vecht CJ. Effect of age on treatment decisions in lowgrade glioma. I Neurol Neurosurg Psychiatry 1993;56: 1256-64.

28 Chamberlain MC, Murovic JA, Levin VA. Absence of contrast enhancement on CT brain scans of patients with supratentorial malignant gliomas. Neurology 1988;38: 1371-4.

\section{NEUROLOGICAL STAMP}

\section{Gregor Johann Mendel (1822-84)}

The Austrian monk, Gregor Johann Mendel, discovered the basic laws of heredity and laid the foundation for the science of modern genetics. The importance of his work was not recognised until 1900, 12 years after Mendel's death and 34 years after its publication. His childhood experience of horticultural work as the son of a peasant farmer had given him an interest in the role of hybrids in evolution. Much of his work was performed on the edible pea, which he grew in the monastery garden. Between 1856 and 1863 he cultivated and tested at least 28000 pea plants, carefully analysing pairs of contrasting traits (such as plant tallness, flower colour, seed shape, pod shape, and flower position on the stem) that appeared in different pea plant progeny. From his findings, Mendel concluded that each plant contributed a factor that determines a particular trait and that pairs of factors in the offspring do not give rise to an amalgamation of traits. These conclusions, in turn, led him to formulate his laws of segregation and the law of independent assortment of characters, which are now recognised as two of the fundamental laws of heredity.

He had entered the Augustine order of Brünn in 1843, where he was ordained in 1847, and he was a science student at the University of Vienna from 1851-53. Mendel reported his findings to the Brünn Society for natural science in 1865 and in the following year published experiments with plant hybrids.

He noted that various traits were inherited as separate units, each of which were inherited independently of the others. He suggested that each parent has pairs of units but only one unit from each pair contributed to the offspring. The units that Mendel described were later named genes. The word was coined in 1906 by the British biologist William Batson.

Mendel had read a brief account of his research to the Brünn Natural History Society in 1865 and asked members to extend his methods to other species, but none did. In 1866 he published his work in the Society's Verhandlungen, a journal distributed to 134 scientific institutions and he also sent reprints of the paper to hybridisation

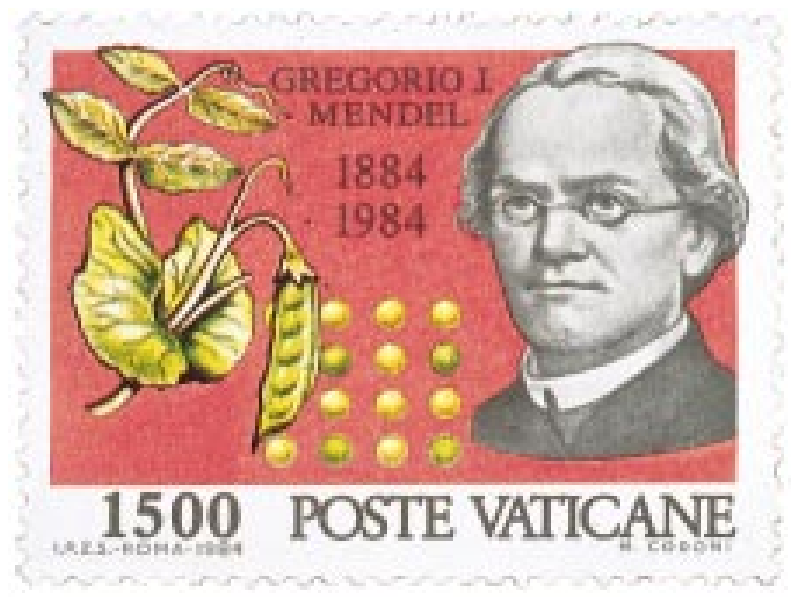

experts of the time. His article seemed to have no effect whatever on the biological thinking of the time in Brünn or elsewhere despite his publications reaching the major libraries of Europe and America. It was not until 1900 when his work was rediscovered by several plant breeders independently that its true value was realised. In 1900, three investigators, K E Correns of Germany, E Tshermak von Seysenegg of Austria, and Hugo de Vries of The Netherlands independently obtained results similar to Mendel's and after searching the scientific literature, located his original reports. Fame only came to him after his death.

Mendel became Abbot of a monastery in 1868 and with its host of administrative duties found less time to devote to his research. By 1870 he had ceased his experiments on heredity. He was honoured philatelically on several occasions. Here he is shown on a stamp by the Vatican in 1984 (Stanley Gibbons 807, Scott 732 ) on the centenary of his death from chronic glomerulonephritis. The illustration shows the inherited characteristics of yellow and green seeds. After his death his associates placed his well bound books in a monastery library and burnt everything else. Thus the original records of his work were lost except for two brief publications rediscovered in 1900 .

L F HAAS 